\title{
A Markov chain Monte Carlo analysis to constrain dark matter properties with directional detection
}

\author{
J. Billard * F. Mayet, and D. Santos \\ Laboratoire de Physique Subatomique et de Cosmologie, \\ Université Joseph Fourier Grenoble 1, CNRS/IN2P3, \\ Institut Polytechnique de Grenoble, Grenoble, France
}

(Dated: October 3, 2018)

\begin{abstract}
Directional detection is a promising dark matter search strategy. Indeed, weakly interacting massive particle (WIMP)-induced recoils would present a direction dependence toward the Cygnus constellation, while background-induced recoils exhibit an isotropic distribution in the Galactic rest frame. Taking advantage of these characteristic features and even in the presence of a sizeable background, it has recently been shown that data from forthcoming directional detectors could lead either to a competitive exclusion or to a conclusive discovery, depending on the value of the WIMP-nucleon cross section. However, it is possible to further exploit these upcoming data by using the strong dependence of the WIMP signal with : the WIMP mass and the local WIMP velocity distribution. Using a Markov chain Monte Carlo analysis of recoil events, we show for the first time the possibility to constrain the unknown WIMP parameters, both from particle physics (mass and cross section) and Galactic halo (velocity dispersion along the three axis), leading to an identification of non-baryonic dark matter.
\end{abstract}

PACS numbers: 95.35.+d, 14.80.-j

\section{INTRODUCTION}

Directional detection of Galactic dark matter has been first proposed by D. N. Spergel [1] highlighting the fact that even low angular resolution directional detectors could be used to show a clear asymmetry in the forward/backward distribution of weakly interacting massive particle (WIMP) events with respect to the direction of the Cygnus constellation.

Beyond the simple asymmetry feature, it has recently been shown that dedicated statistical data analysis of forthcoming directional detectors [2-6] could lead either to a competitive exclusion [7] or to a conclusive discovery [8, 9], depending on the value of the WIMP-nucleon cross section. In the latter case, by using a map based likelihood analysis and even in the presence of a sizeable background, it is possible to show that the main incoming direction does correspond to the direction of the Cygnus constellation $\left(\ell_{\odot}, b_{\odot}\right)$. This is indeed the discovery proof of this detection strategy and it has been shown that a $10 \mathrm{~kg} \mathrm{CF} 4$ detector (MIMAC) operated during 3 years, would allow for a high significance discovery down to $\sigma^{S D} \simeq 10^{-4} \mathrm{pb}[10]$. In this paper, we strive to go one step beyond by trying to constrain the properties of Galactic dark matter with directional detection.

Indeed, constraining WIMP parameters (mass $m_{\chi}$ and cross section $\sigma_{n}$ ) with upcoming dark matter experiments is a main concern of current phenomenological studies, using either indirect detection [11, 12],

*Electronic address: billard@lpsc.in2p3.fr direct detection on its own [12 17], in combination with collider data 19 or with the measurements of halo star kinematics 20]. The quest for a modelindependent formalism is a difficult task as the signal expected in direct detection depends on the properties of both the WIMP particle (mass and cross section) and the Galactic dark matter halo (three-dimensional local WIMP velocity distribution and density). This approach is of particular interest in the context of competitive upcoming experiments which might be able to give positive WIMP detection instead of background rejection. M. Drees and C. L. Shan have proposed a model-independent reconstruction of the WIMP velocity distribution as well as its various moments (mean velocity, dispersions, ...), providing the WIMP mass is $a$ priori known [15] or deduced from positive signals from at least two direct detectors with different target nuclei [16]. The complementary approach is to constrain the WIMP properties with the help of a high dimensional multivariate analysis and within the framework of a general halo model, with a large number of parameters. Thus, the main strength of this study, and hence of directional detection, is the possibility of constraining the properties of both the dark matter particle and the dark matter halo with a single experiment. The choice of the fitting model must be well motivated e.g. by $\mathrm{N}$-body simulations, as it remains as an ansatz.

Directional detection presents a high identification potential thanks to the use of the double-differential spectrum $\mathrm{d}^{2} R / \mathrm{d} E_{R} \mathrm{~d} \Omega_{R}$, also called the directional event rate, in a given recoil energy range. Indeed, its shape depends both on the WIMP mass and WIMP velocity distribution, while the magnitude mainly depends on the product of the local WIMP density 
and the WIMP-nucleon cross section. Within the framework of a multivariate recoil event analysis using a Markov chain Monte Carlo (MCMC), we show for the first time the possibility to constrain, with a single directional experiment, the unknown WIMP parameters, both from particle physics $\left(m_{\chi}, \sigma\right)$ and Galactic halo (velocity dispersion along the three axis), leading to an identification of non-baryonic dark matter. It is, of course, possible to include external data, e.g. halo star kinematics as in 20], and to relax some astrophysical inputs, as $\rho_{0}$ for instance. However, in this work, we focus on the contribution of directional detection on its own, highlighting the need for future large directional detectors.

The paper is organized as follows. In Sec. II, the dark matter halo modeling is introduced while the directional detection framework is presented in Sec. III. Then, the Markov chain Monte Carlo analysis is detailed in Sec. IV, highlighting the performance of such a method in the context of directional detection. Sec. V presents the results of this 8 parameter analysis for a directional detector with a sizeable background contamination and in the case of a benchmark dark matter model. Departures from this input model, by changing the WIMP mass, the velocity anisotropy and the background assumptions are presented in Sec. VI.

\section{DARK MATTER HALO MODELING}

Direct detection depends crucially on the local WIMP velocity distribution 21 23 and it is important to investigate the effect of halo modeling on exclusion limits and allowed regions. The alternative strategy is to build a multivariate analysis, using a halo model with a large number of parameters to be constrained by the analysis. The isothermal sphere halo model is often considered but it is worth going beyond the standard assumption especially when considering recent hints in favor of triaxiality.

Indeed, recent results from N-body simulations are in favor of triaxial dark matter halos with anisotropic velocity distributions and potentially containing substructures as subhalos (clumps) and dark disk [24 27]. Moreover, recent observations of Sagittarius stellar tidal stream have shown evidence for a triaxial Milky Way dark matter halo [28], with the short axis being approximately aligned with the Galactic $\hat{x}$ axis (toward the Galactic center), and the longest with the Galactic $\hat{y}$ axis (in the direction of the solar motion). However, it is noteworthy that this result holds true at large radius $(60$ $\mathrm{kpc}$ ) and N-body simulations have shown that there can be significant variations of the axis ratios with radius [29]. Hitherto, there is no observational evidence of triaxiality at solar position.

The multivariate Gaussian WIMP velocity distribution has been first proposed by N. W. Evans et al. [30]. It corresponds to the simplest triaxial generalization of the standard isothermal sphere with a density profile $\rho(r) \propto$ $1 / r^{2}$, leading to a smooth WIMP velocity distribution without substructure, with a flat rotation curve and in dynamical equilibrium. The velocity dispersion tensor $\boldsymbol{\sigma}_{v}$, given by the Jeans equations, is symmetric. Thus, one can find an orthogonal basis in which the tensor is diagonal leading to the following expression of the WIMP velocity distribution in the solar system rest frame,

$$
f(\vec{v})=\frac{1}{\left(8 \pi^{3} \operatorname{det} \boldsymbol{\sigma}_{v}^{2}\right)^{1 / 2}} \exp \left[-\frac{1}{2}\left(\vec{v}-\vec{v}_{\odot}\right)^{T} \boldsymbol{\sigma}_{v}^{-2}\left(\vec{v}-\vec{v}_{\odot}\right)\right]
$$

where the velocity dispersion tensor $\boldsymbol{\sigma}_{v}=\operatorname{diag}\left[\sigma_{x}, \sigma_{y}, \sigma_{z}\right]$ is assumed to be diagonal in the Galactic rest frame and $\vec{v}_{\odot}$ is the Sun motion with respect to the Galactic rest frame.

The velocity anisotropy $\beta(r)$, is then defined as 31],

$$
\beta(r)=1-\frac{\sigma_{y}^{2}+\sigma_{z}^{2}}{2 \sigma_{x}^{2}}
$$

According to N-Body simulations with or without baryons [25, 32 34], the $\beta$ parameter at $R_{\odot}=8 \mathrm{kpc}$ of the Galactic center, spans the range $0-0.4$ which is in favor of radial anisotropy.

As stated above, our choice is to develop a high dimensional multivariate analysis considering a general enough halo model, i.e. with a large number of parameters, and by constraining all of them with the data analysis of a single experiment. The choice of the halo model must be carefully done as it remains as an ansatz. Following recent results from N-body simulations with baryons [25, 35], the choice of a multivariate Gaussian seems to be a reasonable guess, although one could argue that deviations are observed in the WIMP velocity distribution, making it closer to a generalized Gaussian or even a double Maxwellian distribution when considering the presence of a corotating dark disk. We argue that worrying about the exact shape of the WIMP velocity distribution seems to be not relevant, in particular when taking into account the fact that the resolution of current numerical simulations is many orders of magnitude larger than the scale of the ultralocal dark matter distribution probed by current and future detectors. This is why we have chosen a multivariate Gaussian WIMP velocity distribution as a fitting model, in a first attempt to constrain both the WIMP parameters $\left(m_{\chi}, \sigma_{n}\right)$ and the dark matter halo properties using directional detection. Effect of nonsmooth halo model with substructures and/or streams will be addressed in a forthcoming paper.

In the following, the input halo model used to generate simulated data, is chosen according to two models : a standard isotropic halo $(\beta=0)$ in which case the velocity dispersions are linked to the local circular velocity $v_{0}=220 \mathrm{~km} / \mathrm{s}$ as $\sigma_{x}=\sigma_{y}=\sigma_{z}=v_{0} / \sqrt{2} \approx 155$ 
$\mathrm{km} / \mathrm{s}$; and an anisotropic halo $(\beta=0.4)$, with the following velocity dispersions $\left\{\sigma_{x}=200 \mathrm{~km} / \mathrm{s} ; \sigma_{z}=169\right.$ $\left.\mathrm{km} / \mathrm{s} ; \sigma_{y}=140 \mathrm{~km} / \mathrm{s}\right\}$. The latter case corresponds to the logarithmic ellipsoidal halo model from [30] with the Sun located on the major axis of the halo, the axis ratios $p$ and $q$ being equal to 0.9 and 0.8 , respectively. This is usually taken as an extreme case for the anisotropy, in order to avoid instabilities arising when the ratio of any of the velocity dispersion is greater than 3 . Indeed, as discussed in [22, 30], in order to consider only physically relevant model, every velocity dispersions has to satisfy the following constraint: $\sigma_{j} / 3<\sigma_{i}<3 \sigma_{j}$.

\section{DIRECTIONAL DETECTION FRAMEWORK}

\section{A. Detector configuration}

Several dark matter directional detectors [2] are being developed and/or operated : MIMAC [3], DRIFT [4], DM-TPC [5] and NEWAGE [6]. Directional detection requires 3D track reconstruction of recoiling nuclei down to a few $\mathrm{keV}$ with sense recognition. In fine, an ideal directional detector should allow one to evaluate the double-differential spectrum $\mathrm{d}^{2} R / \mathrm{d} E_{R} \mathrm{~d} \Omega_{R}$ in a given recoil energy range $\left[E_{R_{1}}, E_{R_{2}}\right]$. The lower bound is due to the threshold ionization energy taking into account the quenching factor, while the upper bound allows one to limit background contamination, as most of the WIMP events are concentrated at low-recoil energy.

In the following, we consider an ideal detector configuration which could be within reach in a few years. The configuration of the MIMAC project is chosen : a $10 \mathrm{~kg}$ $\mathrm{CF}_{4}$ detector, operated at $50 \mathrm{mbar}$ and allowing $3 \mathrm{D}$ reconstruction of recoiling tracks with sense recognition. The chosen recoil energy range is between 5 and $50 \mathrm{keV}$ and an exposure $\xi=30 \mathrm{~kg}$.year is taken into account for data simulation. In order to treat realistic cases, we allow for a sizeable residual background contamination in the data. Indeed, the discrimination of isotropic background events from WIMP events has been early recognized as the main strength of this detection strategy [1]. However, as discussed in $7,8,12,14,16,17]$, one of the key issues for direct detection is the unknown background energy distribution. Two extreme cases may be considered [12 14, 16, 17] : flat or exponentially decreasing with increasing recoil energy, i.e. with the same feature as the WIMP-induced energy spectrum. Within the framework of a dedicated statistical data analysis aiming at the identification of dark matter, residual background should be accounted for and we will show that it does only mildly alter the result.

Energy and angular resolutions are other points to be carefully handled. However, it depends on various track parameters such as track length, gaz mixture, initial track position and direction. A full study of 3D track reconstruction is underway [18] and we argue that taking into account finite angular resolution required a full coupling of track reconstruction analysis with this MCMC method. As a first step, and as our goal is to show the identification potential of directional detection, an ideal detector is considered hereafter, i.e. perfect energy and angular resolutions. As shown in 7], the effect of finite angular/energy resolution has been shown to be small as far as directional exclusion limits are concerned, providing the angular resolution is well estimated via detector commissioning, e.g. by using a neutron field [36].

\section{B. Directional detection}

The detector velocity in the Galactic rest frame corresponds to $\vec{v}_{\odot}$, when neglecting the Sun peculiar velocity and the Earth orbital velocity about the Sun 52]. We consider the value $\vec{v}_{\odot}=220 \mathrm{~km} \cdot \mathrm{s}^{-1}$ along the $\hat{y}$ axis. In such case, the main incoming direction of the WIMP signal should be pointing toward $\left(\ell_{\odot}=90^{\circ}, b_{\odot}=0^{\circ}\right)$. Using the Galactic coordinates $(\ell, b)$, the WIMP velocity is written in the Galactic rest frame as:

$$
\vec{v}=v(\cos \ell \cos b \hat{x}+\sin \ell \cos b \hat{y}+\sin b \hat{z})
$$

Following [37], the directional recoil rate is given by,

$$
\frac{\mathrm{d}^{2} R}{\mathrm{~d} E_{R} \mathrm{~d} \Omega_{R}}=\frac{\rho_{0} \sigma_{0}}{4 \pi m_{\chi} m_{r}^{2}} F^{2}\left(E_{R}\right) \hat{f}\left(v_{\min }, \hat{q}\right),
$$

with $m_{\chi}$ the WIMP mass, $m_{r}$ the WIMP-nucleus reduced mass, $\rho_{0}=0.3 \mathrm{GeV} / \mathrm{c}^{2} / \mathrm{cm}^{3}$ the local dark matter density (see Sec. VC for discussion), $\sigma_{0}$ the WIMP-nucleus elastic scattering cross section, $F\left(E_{R}\right)$ the form factor, $\hat{q}$ refers to the recoil direction expressed in the Galactic coordinates and $v_{\min }=\sqrt{m_{N} E_{R} / 2 m_{r}^{2}}$ is the minimal WIMP velocity required to produce a nuclear recoil of energy $E_{R}$. In the case of an axial coupling and within the Born approximation, the expression of the form factor is given by [38] :

$$
F\left(E_{R}\right)=\frac{\sin \left[\sqrt{2 m_{N} E_{R}} \times R\left({ }^{A} X\right)\right]}{\sqrt{2 m_{N} E_{R}} \times R\left({ }^{A} X\right)}
$$

where $R\left({ }^{A} X\right)$ is the radius of the target nucleus.

Eventually, $\hat{f}\left(v_{\min }, \hat{q}\right)$ is the three-dimensional Radon transform [39] of the WIMP velocity distribution $f(\vec{v})$ defined as,

$$
\hat{f}\left(v_{\min }, \hat{q}\right)=\int d^{3} v \delta\left(v_{\min }-\vec{v} \cdot \hat{q}\right) f(\vec{v})
$$

Geometrically, the Radon transform is the integral of the function $f(\vec{v})$ on a plane orthogonal to the direction $\hat{q}$ at a distance $v_{\min }$ from the origin. Using the Fourier slice theorem, P. Gondolo found the expression of the Radon transform of the multivariate Gaussian to be [37],

$$
\hat{f}\left(v_{\min }, \hat{q}\right)=\frac{1}{\left(2 \pi \hat{q}^{T} \boldsymbol{\sigma}_{v}^{2} \hat{q}\right)^{1 / 2}} \exp \left[-\frac{\left[v_{\min }-\hat{q} \cdot \vec{v}_{\odot}\right]^{2}}{2 \hat{q}^{T} \boldsymbol{\sigma}_{v}^{2} \hat{q}}\right] .
$$


Together with (4), this expression is of particular interest in the context of massive MCMC calculations, as it allows one to avoid time-consuming evaluation of the 3D integral of $f(\vec{v})$ for each event at each step. It is, however, equivalent to the directional recoil rate of [40].

In the energy range $\left[E_{R_{1}}, E_{R_{2}}\right]$, the expected number of WIMP events $\mu_{s}$ corresponding to a given set of physical parameters is given by,

$$
\mu_{s}=\xi \int_{E_{R_{1}}}^{E_{R_{2}}} \int_{\Omega_{R}} \frac{\mathrm{d}^{2} R}{\mathrm{~d} E_{R} \mathrm{~d} \Omega_{R}} \mathrm{~d} E_{R} \mathrm{~d} \Omega_{R}
$$

where $\xi$ is the total exposure.

\section{THE DIRECTIONAL MARKOV CHAIN MONTE CARLO METHOD}

We present a new method, based on a Markov chain Monte Carlo analysis, to extract information on dark matter from directional data. First, the interest of use of MCMC algorithm is outlined. Then, the method is fully described in the following section. Discussion on chain efficiency will be done in order to prove that MCMC algorithms are suited for this type of analysis.

\section{A. Interest of the MCMC algorithm}

As stated above, directional detection offers the possibility of using three-dimensional datasets: the recoil energy $E_{R}$ and its direction in Galactic coordinates $\left(\ell_{\mathrm{R}}, \mathrm{b}_{\mathrm{R}}\right)$. It follows that constraints on dark matter properties should be enhanced by the use of directional detection when compared to directional insensitive detection. This is of particular interest when developing a high dimensional multivariate analysis aiming at going beyond the standard isotropic halo assumption. Indeed, the information enclosed in the directional event rate $\left(d^{2} R / d E_{R} d \Omega_{R}\right)$, i.e. the energy spectrum and the $2 \mathrm{D}$ shape of the angular distribution, allows one in principle to increase the number of degrees of freedom of the fitting model. Indeed, adding directional information to the energy one allows one to remove degeneracies among fitting parameters and hence to deduce consistent constraints.

In the following, we list the free parameters of our fitting model :

- $\left(\sigma_{x}, \sigma_{y}, \sigma_{z}\right)$ the three velocity dispersions of the local WIMP velocity distribution,

- $\left(\ell_{\odot}, b_{\odot}\right)$ referring to the main direction of the recoiling nuclei. It is indeed an unambiguous signature of dark matter detection [8],

- $m_{\chi}$ the WIMP mass,
- $\sigma_{n}$ the WIMP-nucleon cross section directly related to $\sigma_{0}$ in the pure proton approximation for the fluorine target,

- $R_{b}$ the background event rate in the considered energy range $[5,50] \mathrm{keV}$. The background events are those remaining after the electron/nuclear recoil rejection, based for instance on the length/energy discrimination [41].

This leads to an eight-parameter analysis of directional dark matter dataset allowing us to quantitatively constrain the WIMP properties and the dark matter halo profile. Prior ranges are presented in Table I

As the number of free parameters is large, grid calculation of likelihood or $\chi^{2}$ functions are not suitable due to the exponential growth of the volume of the parameter space. Indeed, in order to ensure a scan of all the physical parameter space, the regions of interest, i.e. the region where the model fits the data, will fill only a tiny part of the whole volume. This corresponds to a waste of computation time that is avoided using MCMC algorithm. Indeed, Markov chains are used in order to sample the likelihood (or $\chi^{2}$ ) distribution according to Bayesian statistics, enabling the enlargement of the parameter space at a minimal computing time cost by focusing on the regions of interest.

In the following we provide a brief description of the MCMC, emphasizing its use in the context of directional detection. We refer the reader to a more complete description, within the framework of cosmic ray physics, in [42] and references therein.

\section{B. Description of the method}

In a general description, an $m$-dimensional parameter space is described by the following basis $\vec{\theta}=$ $\left\{\theta^{(1)}, \theta^{(2)}, \ldots, \theta^{(m)}\right\}$, where each element $\theta^{(\alpha)}$ refers to one of the physical parameter of interest. The MCMC algorithm enables us to sample the conditional posterior Probability Density Functions (PDF) of each parameter given the data $P(\vec{\theta} \mid \vec{D})$, where $\vec{D}$ refers to the number of

\begin{tabular}{|c|c|}
\hline Parameter & Prior range \\
\hline \hline$m_{\chi}\left(\mathrm{GeV} / \mathrm{c}^{2}\right)$ & $(5,1000)$ \\
\hline $\log _{10}\left(\sigma_{n}(\mathrm{pb})\right)$ & $(-5,-1)$ \\
\hline$\ell_{\odot}\left(^{\circ}\right)$ & $(-180,+180)$ \\
\hline$b_{\odot}\left({ }^{\circ}\right)$ & $(-90,+90)$ \\
\hline$\sigma_{x, y, z}\left({\left.\mathrm{~km} . \mathrm{s}^{-1}\right)}^{-1}\right)$ & $(5,500)$ \\
\hline$R_{b}\left(\mathrm{~kg}^{-1}\right.$ year $\left.^{-1}\right)$ & $(0,50)$ \\
\hline
\end{tabular}

TABLE I: Parameters with their uniform prior ranges used for all MCMC analysis. 
events $N$, their direction $\left(\ell_{R}, b_{R}\right)$ and their energy $E_{R}$. This can be achieved with the use of the Bayes' theorem applied to parameter inference,

$$
P(\vec{\theta} \mid \vec{D})=\frac{P(\vec{D} \mid \vec{\theta}) \times \Pi(\vec{\theta})}{P(\vec{D})}
$$

where $P(\vec{D})$ is the data probability, also called the $e v$ idence, which can be regarded as a normalization factor, $\Pi(\vec{\theta})$ is the prior probability indicating the degree of belief before observing the data. Finally, $P(\vec{D} \mid \vec{\theta})$ corresponds to the likelihood function written $\mathscr{L}(\vec{\theta})$. In this framework, the posterior PDF $P(\vec{\theta} \mid \vec{D})$ is the normalized product of the likelihood function with the priors. Using a Bayesian approach, the posterior PDF of each single parameter $\theta^{(\alpha)}$ is given by the marginalisation of the multidimensional $P(\vec{\theta} \mid \vec{D})$ distribution over the other parameters $\theta^{(\beta \neq \alpha)}$,

$$
P\left(\theta^{(\alpha)} \mid \vec{D}\right)=\int_{\Omega_{\beta}, \forall \beta \in[1, m] \backslash\{\alpha\}} P(\vec{\theta} \mid \vec{D}) \mathrm{d} \theta^{(\beta)} .
$$

From each one-dimensional PDF, we can estimate the expected value of a given parameter and its confidence level (CL). The difficulty is then to evaluate the multidimensional target PDF $p(\vec{\theta}) \equiv P(\vec{\theta} \mid \vec{D})$. For the abovementioned reasons, instead of using a grid calculation algorithm, we developed a MCMC algorithm in order to evaluate $p(\vec{\theta})$. This Monte Carlo sampling of the target function is done using Markov chains which are a sequence of $N$ points in the $m$-dimensional parameter space,

$$
\left\{\vec{\theta}_{i}\right\}_{i=1, \ldots, N} \equiv\left\{\vec{\theta}_{1}, \vec{\theta}_{2}, \ldots, \vec{\theta}_{N}\right\}
$$

which is constructed according to the MetropolisHastings algorithm ensuring that the stationary distribution of each chain corresponds to the target distribution $p(\vec{\theta})$ being sampled. The Metropolis-Hastings algorithm is a random walk in the parameter space where each step $\vec{\theta}_{i+1}$ is derived from the step $\vec{\theta}_{i}$ with the following procedure :

- At each step $\vec{\theta}_{i}$ a trial step $\vec{\theta}_{\text {trial }}$ is generated from a proposal distribution $q\left(\vec{\theta}_{\text {trial }} \mid \vec{\theta}_{i}\right)$.

- This trial step is accepted or not according to the acceptance probability $a$ calculated as follows :

$$
a=a\left(\vec{\theta}_{\text {trial }} \mid \vec{\theta}_{i}\right)=\min \left(1, \frac{p\left(\vec{\theta}_{\text {trial }}\right)}{p\left(\vec{\theta}_{i}\right)} \frac{q\left(\vec{\theta}_{\text {trial }} \mid \vec{\theta}_{i}\right)}{q\left(\vec{\theta}_{i} \mid \vec{\theta}_{\text {trial }}\right)}\right)
$$

The probability for the trial step to be accepted is equal to $a$. Notice that in the case of a symmetric proposal function $q$ we have $q\left(\vec{\theta}_{\text {trial }} \mid \vec{\theta}_{i}\right)=q\left(\vec{\theta}_{i} \mid \vec{\theta}_{\text {trial }}\right)$, which simplifies the expression of eq.(12).

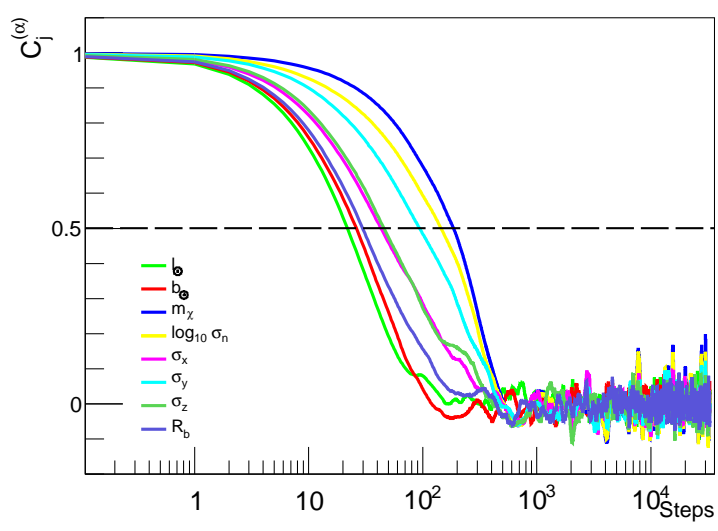

FIG. 1: Correlation function of the 8 parameters from the MCMC run applied to our benchmark model (see sec. V) with the simple multivariate Gaussian proposal function.

- If the trial step is accepted, then $\vec{\theta}_{i+1}=\vec{\theta}_{\text {trial }}$ and if not, the chain stagnate at the same point in the parameter space leading to $\vec{\theta}_{i+1}=\vec{\theta}_{i}$.

Three characteristics of the Markov chains are worth being investigated in order to ensure a consistent sampling of the target function :

Burn-in length (b): it corresponds to the number of steps (or iterations) to be removed from the beginning in order to forget the starting point of the random walk. It is estimated as the first step reaching the median value of the target distribution $E[p(\vec{\theta})]$ as

$$
p\left(\vec{\theta}_{b}\right)>E[p(\vec{\theta})]
$$

Correlation length (l): it is the required minimal length between two steps so that they can be considered as uncorrelated. By construction, each step depends on the previous one. Then, in order to get independent steps, some subsampling is needed. It corresponds to rejecting all steps which are closer than $l$ to each other. The correlation length $l^{(\alpha)}$ of each parameter $\theta^{(\alpha)}$ is estimated by computing the autocorrelation function $c_{j}^{(\alpha)}$ where $j$ corresponds to the distance between two steps. Indeed, $l^{(\alpha)}$ is defined as the smallest $j$ for which the correlation function is strictly less than $1 / 2$, i.e $c_{j}^{(\alpha)}<1 / 2$. It should be noticed that the limit of $1 / 2$ is arbitrary but has been shown to be sufficient in order to consider the steps $\theta_{i}^{(\alpha)}$ and $\theta_{i+j}^{(\alpha)}$ as uncorrelated [42]. Then, the correlation length of the whole chain $l$ is defined as,

$$
l=\max \left[l^{(1)}, \ldots, l^{(\alpha)}, \ldots, l^{(m)}\right]
$$

Hence, in order to consider only independent samples (steps) $\vec{\theta}_{\text {ind }}$ we have subsampled each Markov chain according to the following procedure $\vec{\theta}_{\text {ind }}=\vec{\theta}_{i=b+k l}$ with $k$ 

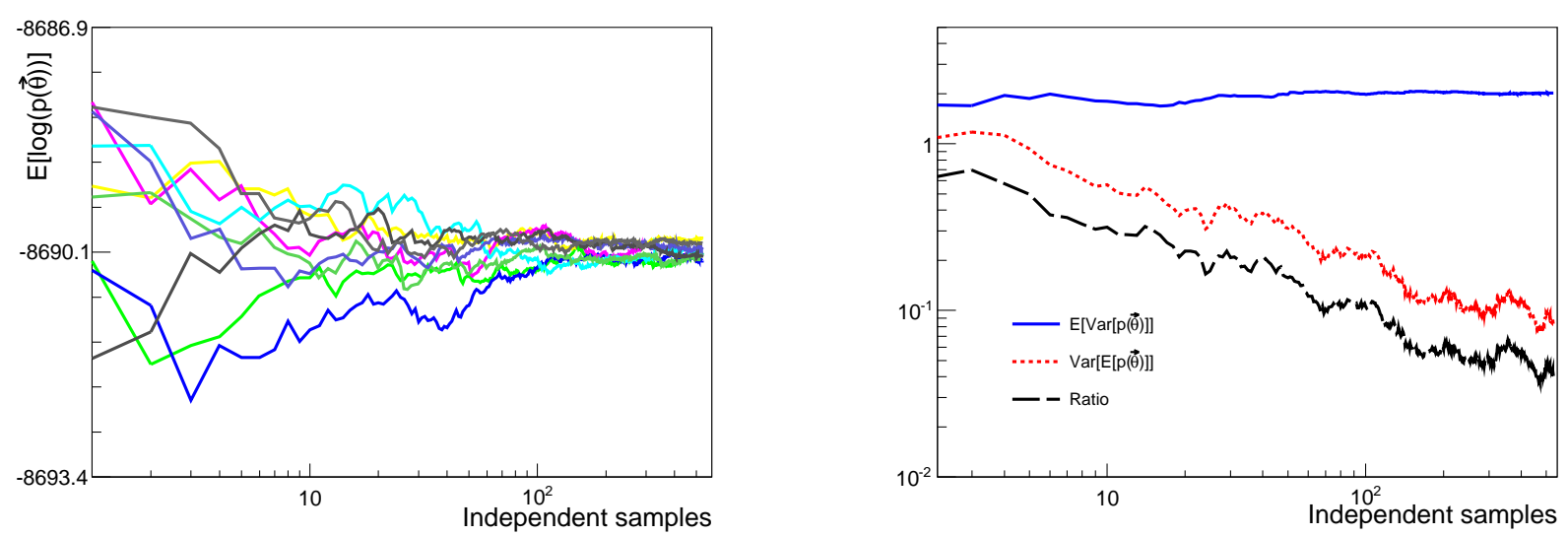

FIG. 2: Left panel : mean of the log-likelihood value of each Markov chain $\mathrm{E}[\log (p(\vec{\theta}))]$ as a function of the number of independent samples for 10 Markov chains. Right panel : $\operatorname{Var}[\mathrm{E}(p(\vec{\theta}))], \mathrm{E}[\operatorname{Var}(p(\vec{\theta}))]$ and convergence ratio $r$ as a function of the number of steps. Both figures come from the MCMC run applied to our benchmark model (see Sec. V).

being an integer. Figure 1 1 represents the autocorrelation function for the eight parameters from a MCMC analysis discussed in Sec. V] For this chain, we can see that the correlation length is equal to 187 due to the WIMP mass parameter. Indeed, as explained in the following section, the strong correlation between the WIMP mass and cross-section will induce larger correlation length. Hence, as the correlation length is linked to the stagnation of the chain, in order to have a smaller value of $l$, the proposal function has to be carefully chosen to approximate the target PDF.

The efficiency of a Markov chain Monte Carlo sampling can then be estimated as the fraction of independent samples $N_{\text {ind }}$ with respect to the total number of samples $N$, where $N_{\text {ind }}$ is given by :

$$
N_{\text {ind }}=\frac{N-b}{l}
$$

More efficient is a MCMC sampling, since the number of rejected samples is lower, leading to a better estimation of the target PDF. The quality of the estimation of the target PDF is directly affected by the MCMC sampling efficiency and hence by the burn-in and correlation lengths. Depending on the input values of the different parameters used to simulate pseudodata of single directional detection experiments, the sampling efficiency is between $0.6 \%$ and $8 \%$. It mainly depends on the correlation lengths which were found to be between 6 and 130 using the second proposal function : the multivariate Gaussian with covariance matrix (see below). The sampling efficiency could be enhanced by using other proposal functions not necessarily Gaussian like the Binary Space Partitioning first introduced in MCMC sampling by A. Putze et al. [42]. However, as we are running a large number of Markov chains in parallel with a low computational time, such efficiencies are largely enough to get well sampled PDFs. chain convergence: it is a key criteria worth being investigated for MCMC sampling as it ensures that the target PDF is being sampled by the different chains. Indeed, the left panel of Fig. 2 presents the evolution of the mean of the log-likelihood value of each Markov chain $\mathrm{E}[\log (p(\vec{\theta}))]$ as a function of the number of independent samples for 10 Markov chains. From this figure, we can appreciate the fact that the mean value of each Markov chain is converging to the same value as well as the variance, not shown here. Then, in order to quantify the convergence, we can form the following ratio :

$$
r=\frac{\operatorname{Var}[\mathrm{E}(p(\vec{\theta}))]}{\mathrm{E}[\operatorname{Var}(p(\vec{\theta}))]}
$$

As seen on the right panel of Fig. 2. $\operatorname{Var}[\mathrm{E}(p(\vec{\theta}))]$ tends to 0 whereas $\mathrm{E}[\operatorname{Var}(p(\vec{\theta}))]$ tends to a finite number leading to $r \rightarrow 0$ when increasing the number of independent samples. Then, we can arbitrarily fix a limit $r_{c}$ which will correspond to a chain convergence if $r<r_{c}$. Following [43], we have chosen $r_{c}=0.2$. Then, from the Fig. 2, we can see that for this MCMC run with 10 independent chains, the convergence status is reached at the $40^{\text {th }}$ independent step (sample). However, even if the chain convergence criteria is reached with only a few tenth of independent samples, one should have longer chains of independent samples to get a very precise estimation of the target function. In our case we have run between 10 and 100 Markov chains of $10^{5}$ steps in order to get more than $5 \times 10^{4}$ independent samples for each analysis. Indeed, another interest in computing several Markov chains in parallel is that we can add all the independent samples from every chain together to enhance the estimation of the target function. 

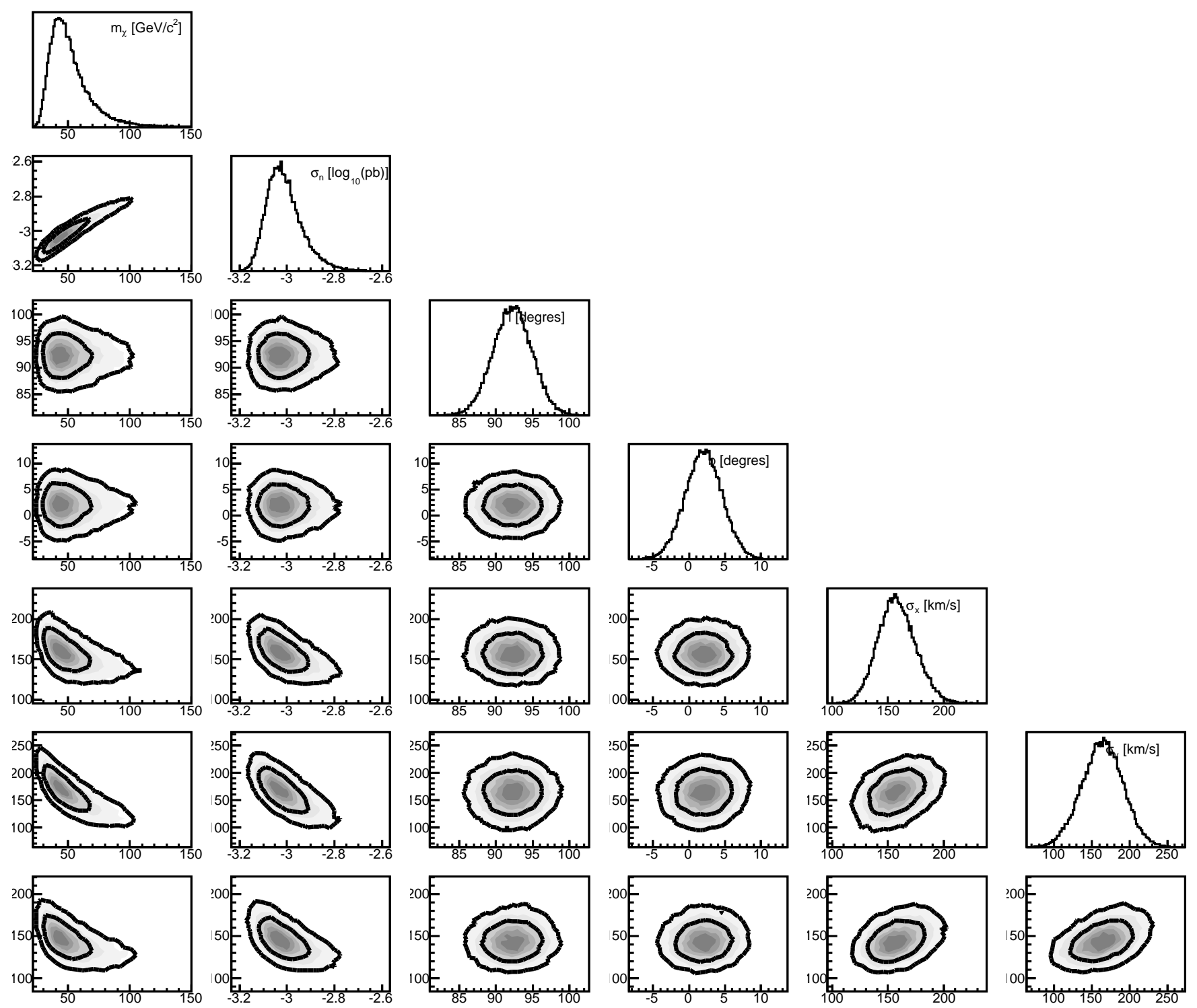

0
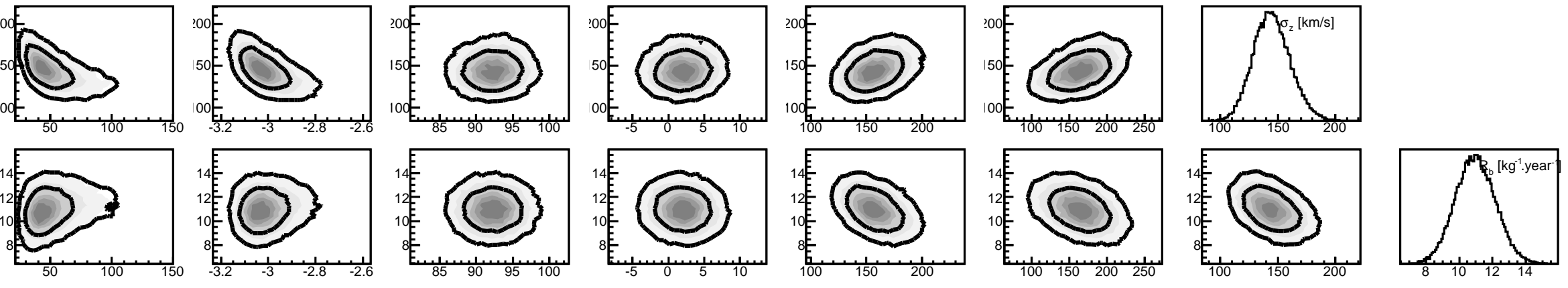
In this paper, we have considered flat prior for each parameter $\left\{m_{\chi}, \log _{10}\left(\sigma_{n}\right), \ell_{\odot}, b_{\odot}, \sigma_{x}, \sigma_{y}, \sigma_{z}, R_{b}\right\}$. In such case, the Bayes' theorem is simplified and the target distribution $p(\vec{\theta})$ reduces to the likelihood function $\mathscr{L}(\vec{\theta})$. The latter is given by the extended likelihood function (see Ref. [44]) dedicated to unbinned data as,

$$
\begin{aligned}
\mathscr{L}(\vec{\theta}) & =\frac{\left(\mu_{s}+\mu_{b}\right)^{N}}{N !} e^{-\left(\mu_{s}+\mu_{b}\right)} \times \\
& \prod_{n=1}^{N_{\text {event }}}\left[\frac{\mu_{s}}{\mu_{s}+\mu_{b}} S\left(\vec{R}_{n}\right)+\frac{\mu_{b}}{\mu_{s}+\mu_{b}} B\left(\vec{R}_{n}\right)\right]
\end{aligned}
$$

where $\mu_{s}$ and $\mu_{b}=R_{b} \times \xi$ are the expected number of WIMP events and background events respectively. $\vec{R}_{n}$ refers to the energy and direction of each event while the functions $S$ and $B$ are the directional event rate of the WIMP events and the background events, respectively.

As previously highlighted, in order to optimize the MCMC sampling efficiency, the proposal function must be as close as possible to the target PDF. Two different successive proposal functions are used :

- A multivariate Gaussian in the same basis of the parameter space with dispersions $\sigma^{(\alpha)}$ taken from a fast evaluation of the likelihood function on a grid. In such case, we have,

$$
\theta_{\text {trial }}^{(\alpha)}=\theta_{i}^{(\alpha)}+\sigma^{(\alpha)} x
$$

where $x$ is a random variable distributed following the normal distribution $\mathscr{N}(0,1)$.

- A multivariate Gaussian with the covariance matrix estimated from the previous run. Then, the next step is calculated using:

$$
\vec{\theta}_{\text {trial }}=\vec{\theta}_{i}+P C \vec{x}
$$

where $C$ is the eigenvalue of the covariance matrix, $P$ is the matrix of the corresponding eigenvectors and $\vec{x}$ is a vector of $m$ random variables distributed following $\mathscr{N}(0,1)$.

In both cases, as the proposal function is a Gaussian, we are in the case where $q\left(\vec{\theta}_{\text {trial }} \mid \vec{\theta}_{i}\right)=q\left(\vec{\theta}_{i} \mid \vec{\theta}_{\text {trial }}\right)$ which simplifies the expression of the acceptance (Eq. 12).

\section{RESULT FOR A BENCHMARK INPUT DARK MATTER MODEL}

For concreteness, we exemplify this directional MCMC method by studying the case of a given benchmark input model, i.e. the standard isothermal sphere with an isotropic velocity distribution with $\beta=0$ (see Sec. II for more details). A sizeable background contamination $\left(10 \mathrm{~kg}^{-1}\right.$ year $\left.^{-1}\right)$ is accounted for, with a flat energy spectrum. We consider a $50 \mathrm{GeV} / \mathrm{c}^{2}$ WIMP with a WIMP-nucleon axial cross section $\sigma_{n}=10^{-3} \mathrm{pb}$. The input model is used to generate simulated data in a $10 \mathrm{~kg} \mathrm{CF}_{4}$ detector (as proposed by the MIMAC collaboration) with a three-year exposition time. These data are then analyzed with the directional MCMC method (Sec. IV). As stated above, the eight parameters $\left\{m_{\chi}, \log _{10}\left(\sigma_{n}\right), \ell_{\odot}, b_{\odot}, \sigma_{x}, \sigma_{y}, \sigma_{z}, R_{b}\right\}$, are taken as free parameters in the MCMC analysis, with flat priors, thus ensuring that the study is model-independent from a point of view of both particle physics (WIMP properties) and Galactic physics (halo properties). In particular, no previous knowledge of the Galactic dark matter halo is needed, the goal being to extract the posterior PDF of all parameters and check their consistencies with the input model. Departure from isotropy as well as modification of the various parameters of the input model will be studied in Sec. VI.

Figure 3 presents marginalized distributions (diagonal) and $2 \mathrm{D}$ correlations (off-diagonal) plots of the eight parameters of the analysis of simulated data obtained with the benchmark input model. The complete result for each parameter, as extracted from marginalized distributions, is summarized in Table II where the output parameters are characterized by the mean value extracted from their 1D posterior PDF, while the error bars are accounted for a $68 \%$ confidence level. However, to fully understand correlations between the parameters, the full set of 2D correlations is needed. Moreover, to quantify those correlations among the eight different parameters, the correlation matrix defined as

$$
\rho^{\alpha, \beta}=\rho\left[\theta^{(\alpha)}, \theta^{(\beta)}\right]=\frac{\operatorname{cov}\left[\theta^{(\alpha)}, \theta^{(\beta)}\right]}{\sqrt{\operatorname{var}\left[\theta^{(\alpha)}\right] \operatorname{var}\left[\theta^{(\beta)}\right]}}
$$

is given in Fig. 14 and will be discussed hereafter.

The result obtained is threefold : the discovery proof is given by the reconstruction of the main incoming direction $\left(\ell_{\odot}, b_{\odot}\right)$ (Sec. $\mathrm{VA}$ ). Then, the three velocity dipersions and hence the velocity anisotropy parameter $(\beta)$ of the dark matter halo are assessed (Sec. VB leading to a constraint on the properties of the WIMP particle on the $\left(m_{\chi}, \log _{10}\left(\sigma_{n}\right)\right)$ plane, within the framework of our ansatz. In the following, we detail the results arising from the analysis of Fig. 3 .

\section{A. Discovery proof}

Following a previous study [8], we first present the extraction of the main incoming direction of the events $\left(\ell_{\odot}, b_{\odot}\right)$, from a pseudodata analysis. This is a blind analysis as these two parameters are taken as free parameters of the analysis. It can be concluded from marginalized distributions of Fig. 3 that the recovered main recoil direction is pointing towards the Cygnus constellation within $2.5^{\circ}$ at $68 \% \mathrm{CL}$, corresponding to a nonambiguous detection of particles from the Galactic halo which is 


\begin{tabular}{|c||c|c|c|c|c|c|c|c|c|}
\hline & $m_{\chi}\left(\mathrm{GeV} / \mathrm{c}^{2}\right)$ & $\log _{10}\left(\sigma_{n}(\mathrm{pb})\right)$ & $\ell_{\odot}\left(^{\circ}\right)$ & $b_{\odot}\left(^{\circ}\right)$ & $\sigma_{x}\left(\mathrm{~km} \cdot \mathrm{s}^{-1}\right)$ & $\sigma_{y}\left(\mathrm{~km} \cdot \mathrm{s}^{-1}\right)$ & $\sigma_{z}\left(\mathrm{~km} \cdot \mathrm{s}^{-1}\right)$ & $\beta$ & $R_{b}\left(\mathrm{~kg}^{-1} \mathrm{year}^{-1}\right)$ \\
\hline \hline Input & 50 & -3 & 90 & 0 & 155 & 155 & 155 & 0 & 10 \\
\hline Output & $51.8_{-19.4}^{+5.6}$ & $-3.01_{-0.08}^{+0.05}$ & $92.2_{-2.5}^{+2.5}$ & $2.0_{-2.5}^{+2.5}$ & $158_{-17}^{+15}$ & $164_{-26}^{+27}$ & $145_{-17}^{+14}$ & $-0.073_{-0.18}^{+0.29}$ & $10.97 \pm 1.2$ \\
\hline
\end{tabular}

TABLE II: Comparison of the values of the parameters for the input model and as extracted after the MCMC analysis from the marginalized distributions. We quote mean value of the PDF distribution and (68\% CL) error bars.

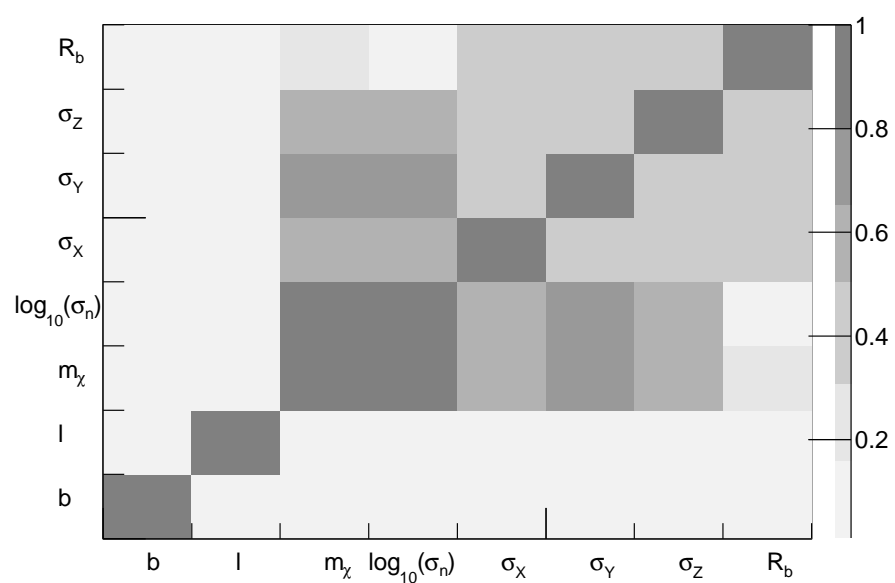

FIG. 4: Correlation matrix as defined by Eq. 20 for the eight parameters of the MCMC analysis in the case of an isothermal halo with a WIMP mass of $50 \mathrm{GeV} \cdot \mathrm{c}^{-2}$ and a WIMP-nucleon cross section. The grey scale represents the absolute values of $\rho^{\alpha, \beta}$. Signs of correlation can be deduced from Fig. 3 .

in favor of a dark matter positive detection.

We found the same result as in [8], which was expected as the information on the main incoming direction is enclosed mainly in the angular part of the WIMP-induced spectrum. The major update is the fact that we prove that this result is model-independent as no a priori knowledge of neither the Galactic dark matter halo nor the WIMP particle is needed. The 2D correlations of Fig. 3 (third and fourth columns and lines) and the two first columns of the correlation matrix (see Fig. (4) indicate that there is no correlation of the main incoming direction with the six other parameters.

In addition, this result holds true for all cases studied hereafter where we have considered different input values of the WIMP mass or halo model. Indeed, for each case, we have checked that the main incoming direction is always consistently constrained and reveals no correlation with other parameters (see Sec. VI).

We emphasize conclusions from [8, 9] : directional detection of dark matter is a powerful strategy to clearly identify a positive dark matter signal, using the main incoming direction as the discovery proof, even in the case of a sizeable background contamination and non standard halo model.

\section{B. Dark matter halo properties}

The originality of this work, in comparison to current phenomenological studies, is that the properties of the dark matter halo itself are constrained using a single directional detection experiment. As shown on Fig. 3, the velocity dispersions are strongly and consistently constrained according to the input values. Indeed, from the marginalized distributions of the posterior PDF of each velocity dispersion, the following constraints can be deduced

$$
\begin{aligned}
& \sigma_{x}=158_{-17}^{+15} \mathrm{~km} \cdot \mathrm{s}^{-1}(68 \% \mathrm{CL}), \\
& \sigma_{y}=164_{-26}^{+27} \mathrm{~km} \cdot \mathrm{s}^{-1}(68 \% \mathrm{CL}), \\
& \sigma_{z}=145_{-17}^{+14} \mathrm{~km} \cdot \mathrm{s}^{-1}(68 \% \mathrm{CL}),
\end{aligned}
$$

giving, in such case, strong evidence in favor of an isotropic dark matter halo.

However, one could notice, from the full MCMC result (Fig. 3) and from the correlation matrix (Fig. 4), that the three velocity dispersions are quite correlated to each other, to the WIMP properties $\left(m_{\chi}, \log _{10}\left(\sigma_{n}\right)\right)$ and to the background rate. In the following, we propose a short discussion to understand the fundamental origin of these different correlations. To begin with, the positive correlation between each of the three velocity dispersions mainly comes from the information on the angular distribution. Indeed, in order to reproduce the shape of the velocity distribution, which is isotropic in this case, the three velocity dispersions have to be positively correlated to each other; in this case, we found $\rho\left[\sigma_{i}, \sigma_{j}\right] \approx 0.4$ for $i \neq j \in\{\mathrm{x}, \mathrm{y}, \mathrm{z}\}$. However, increasing the velocity dispersions leads to an increase in the number of expected WIMP events $\mu_{s}$ and to wider WIMP event angular distribution. The latter can be compensated by decreasing the WIMP mass as it leads to tighter angular distribution (see [7, 8] for a detailed discussion) thus implying a negative correlation between the WIMP mass and the three velocity dispersions with $\rho\left[m_{\chi}, \sigma_{x}\right]=\rho\left[m_{\chi}, \sigma_{z}\right] \approx-0.55$ and $\rho\left[m_{\chi}, \sigma_{y}\right] \approx-0.75$. As the cross section is directly proportional to $\mu_{s}$ the correlations between the $\log _{10}\left(\sigma_{n}\right)$ and the three velocity dispersions are obviously negative with $\rho\left[\log _{10}\left(\sigma_{n}\right), \sigma_{x}\right]=\rho\left[\log _{10}\left(\sigma_{n}\right), \sigma_{z}\right] \approx-0.57$ and $\rho\left[\log _{10}\left(\sigma_{n}\right), \sigma_{y}\right] \approx-0.70$. Correlations between the WIMP parameters and $\sigma_{y}$ are stronger than in the case of $\sigma_{x}$ and $\sigma_{z}$ as it is the most related to $\mu_{s}$ and the total 


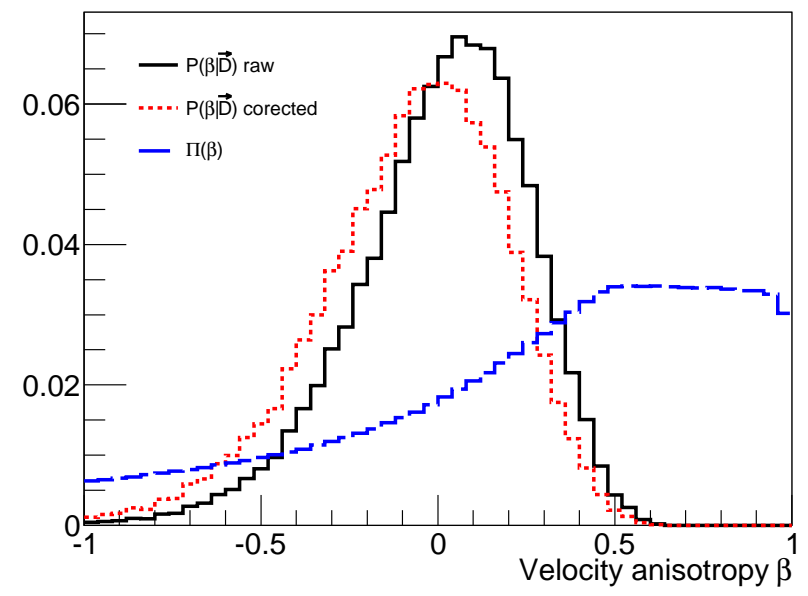

FIG. 5: Posterior PDF distribution of the $\beta$ parameter, with and without correction due to nonflat prior. The prior is Monte Carlo estimated.

width of the angular event distribution. Then, as the velocity dispersion along the $y$ axis is more degenerated with the other parameters than $\sigma_{x}$ and $\sigma_{z}$ the error bar on the estimation of $\sigma_{y}$ are larger than for the two other velocity dispersions (about 2 times larger). The negative correlation between the three velocity dispersions and the background rate can be easily explained by the definition of the extended likelihood function where the sum of $\mu_{s}$ (proportional to the velocity dispersions) with $\mu_{b}$ (proportional to the background rate) follows a Poisson distribution of mean equal to $\mu_{s}+\mu_{b}=N_{\text {event }}$; we found in this case: $\rho\left[R_{b}, \sigma_{j}\right] \approx-0.4$ with $j \in\{\mathrm{x}, \mathrm{y}, \mathrm{z}\}$.

The evaluation of the velocity anisotropy parameter $\beta$ allows us to summarize the results from the three velocity dispersions. Indeed, the posterior PDF of the $\beta$ parameter can be computed from Eq2 However, having a flat prior on the three velocity dispersions implies a nonflat (informative) prior on the $\beta$ parameter. Hence, Fig. 5 presents the raw PDF of $\beta$ considering flat priors on the $\sigma_{i}$ 's in the black solid line, the induced prior on the $\beta$ parameter $\Pi(\beta)$ is shown as the blue dashed line, and the red dotted line corresponds to the corrected PDF of $\beta$ with a flat prior. In the following, for each case, we will only consider the corrected posterior PDF of the $\beta$ parameter. From the latter, we can deduce an interesting constraint $\beta=-0.073_{-0.18}^{+0.29}(68 \%$ CL) favoring an isotropic dark matter halo. This is a proof that within the framework of the multivariate Gaussian halo model, a dedicated MCMC analysis of directional data would allow us to constrain the velocity dispersions, resulting in a discrimination between various halo models.

\section{WIMP parameters}

As stated above, this MCMC analysis also allows us to constrain the parameters of the WIMP by considering both the angular and the energy information from each recoiling event. Figure 3 (first 2 columns) presents marginalized distributions and 2D correlation plots concerning the WIMP parameters $\left(m_{\chi}, \log _{10}\left(\sigma_{n}\right)\right)$. First, we can notice that this analysis method allows us to get satisfactory results, i.e. constraints which are consistent with the input values and with a rather small dispersion:

$$
\begin{gathered}
m_{\chi}=51.8_{-19.4}^{+5.6} \mathrm{GeV} / \mathrm{c}^{2}(68 \% \mathrm{CL}), \\
\log _{10}\left(\sigma_{n}\right)=-3.01_{-0.08}^{+0.05}(68 \% \mathrm{CL})
\end{gathered}
$$

Moreover, as the velocity dispersions are set as free parameters, induced bias due to wrong halo model assumptions is avoided as long as the input halo model is consistent with our ansatz. We refer the reader to 13] for a detailed discussion about the effect of halo model uncertainties on allowed regions. In fact, the combined use of angular and energy information allows us to remove degeneracies amongst the eight parameters and hence to obviate bias in the determination of the WIMP properties.

We observe the usual strong correlation $\rho\left[m_{\chi}, \log _{10}\left(\sigma_{n}\right)\right] \approx 1$ (see Fig. 4) between $m_{\chi}$ and $\log _{10}\left(\sigma_{n}\right)$ which is inherent in the very definition of the event rate, as it scales basically with $\sigma_{n} / m_{\chi}$ for low mass target. We also found a small and positive correlation between the WIMP mass (inversely proportional to $\mu_{s}$ ) and the background rate (proportional to $\left.\mu_{b}\right)$ such as $\rho\left[m_{\chi}, R_{b}\right] \approx 0.25$. Indeed, as mentioned before, this correlation is straightforwardly due to the relationship between $\mu_{s}$ and $\mu_{b}$ where the total number of recorded events follows a Poisson distribution of mean $\mu_{s}+\mu_{b}=N_{\text {event }}$. Finally, we found no correlation between $\log _{10}\left(\sigma_{n}\right)$ and the background rate $R_{b}$.

As a conclusion, directional detection provides a unique opportunity to constrain, with a single experiment, the WIMP mass and the WIMP-nucleon cross section within the framework of a high-dimensional multivariate analysis. This is of great interest in the context of phenomenological efforts [12-17, 19, 45 47] trying to constrain the WIMP parameters $\left(m_{\chi}, \sigma_{n}\right)$ with upcoming dark matter experiments, with either an indirect, direct, or directional strategy. In this work, we have gone one step further in constraining the local WIMP velocity distribution with a single directional detection experiment. It is, of course, possible to include external data as nuisance parameters, e.g. measurement of the local dark matter density $\rho_{0}$ [48, 49], the local circular velocity $v_{0}$ and the escape velocity (taken as infinity in this study). However, it seems premature at the level of a methodological study aimed at showing how to handle directional detection data. For instance, the local WIMP density is usually quoted within the range 
$\rho_{0} \sim 0.2-0.8 \mathrm{GeV} \cdot \mathrm{c}^{-2} \cdot \mathrm{cm}^{-3}$ and we used the so-called "standard" value $0.3 \mathrm{GeV} \cdot \mathrm{c}^{-2} \cdot \mathrm{cm}^{-3}$ for the sake of comparison with various direct detector results [48]. We note that recently, a value of the local dark matter density, $\rho_{0}=0.43 \pm 0.11 \pm 0.10 \mathrm{GeV} \cdot \mathrm{c}^{-2} \cdot \mathrm{cm}^{-3}$, has been derived within the framework of a Galaxy-model-independent method [49]. All constraints on the WIMP-nucleon cross section can be relaxed into constraints on $\rho_{0} \times \sigma$.

\section{Background estimation}

The background rate estimation is also a key point of this analysis strategy, not for the value itself but for the fact that a wrong background estimation may induce bias for other parameters. Indeed, as upcoming data will necessarily be contaminated by some background events, it is important to be able to manage them. As shown in Fig. 3 (last row), it is correctly estimated from the MCMC : $R_{b}=10.97 \pm 1.2 \mathrm{~kg}^{-1}$ year $^{-1}(68 \% \mathrm{CL})$, with tiny correlations with other parameters already discussed in the previous sections. Then, the fact that the background rate is left as a free parameter and reconstructed with the MCMC method allows us to avoid bias in the estimation of the other parameters. Qualitatively, the background rate is mainly constrained by the angular part of the spectrum, more precisely in the hemisphere opposite to the Cygnus constellation, where few WIMP events are expected. In fact, the quality of the estimation of the WIMP and halo parameter is directly related to the estimation of the background rate. In this example, we have shown that dark matter parameter estimation (main direction, WIMP, and dark matter halo properties) is not affected by a rather large background fraction $(\sim 30 \%)$. Hence, directional detection can accommodate to a sizeable background contamination (posterior to data selection), suggesting the idea that light shielding might be sufficient, thus allowing us to reduce muon-induced neutron background [50].

As stated above, for this example a flat background energy spectrum has been considered, which is indeed an optimistic case. In Sec. VI we study the effect of considering an energy distributions for background events which is similar to the one for WIMP events.

\section{RESULTS FOR VARIOUS INPUT MODELS}

The constraints on the different parameters obviously depend on the input model, characterized by the WIMP and dark matter halo properties as well as the background energy spectrum. Indeed, the directional WIMP event rate crucially depends on the dark matter parameters, both from particle physics and Galactic halo physics, and degeneracies may arise depending on their input values. In the following, we explore various input models in order to evaluate their impact on the different constraints which could be obtained with a single

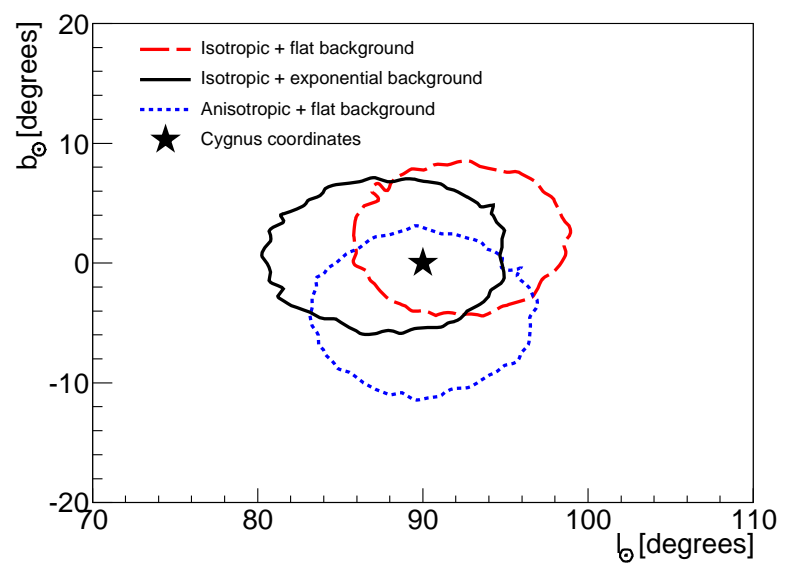

FIG. 6: $95 \%$ contour level in the $\left(\ell_{\odot}, b_{\odot}\right)$ plan for three input models: Isotropic halo model + exponential background (solid line), Isotropic halo model + flat background (long dashed line) and anisotropic halo model + flat background (dotted line).

directional detection experiment, as the one proposed by the MIMAC collaboration, using our MCMC analysis.

The first point worth emphasizing is the fact that in all cases presented hereafter, the recovered main recoil direction is always pointing towards Cygnus, within at most $\sim 4^{\circ}$ at $95 \%$ CL (see Fig. 6). This is relatively straightforward, given the fact that this directional signature is uncorrelated with the other parameters of the MCMC analysis, as emphasized in Sec. VA Indeed, it has been shown in [8] that this directional signature only depends on the background contamination, which is taken equal to $10 \mathrm{evts} / \mathrm{kg} / \mathrm{year}$ in every following cases. This outlines the robustness of the choice of this parameter as a relevant observable to prove that a positive detection of dark matter has been reached by a directional detector. As outlined in [8], this would allow directional detection to provide evidence in favor of a detection of Galactic dark matter even at low exposure and even with a sizeable background contamination. In this study, we have checked that this conclusion holds true even in the case of non standard dark matter halo model.

\section{A. Varying the input WIMP mass}

As highlighted by several previous studies 12 14, 19], the WIMP mass plays a key role in the shape of the allowed regions. We have simulated three different sets of directional data corresponding to an input WIMP mass of $m_{\chi}=20,50,100 \mathrm{GeV} / \mathrm{c}^{2}$ with a constant WIMP-nucleon cross section $\sigma_{n}=10^{-3} \mathrm{pb}$, considering a MIMAC-like directional detector (Sec. IIIB) and the standard isotropic halo model. The results from the three MCMC runs are illustrated in Fig. 7 We present 

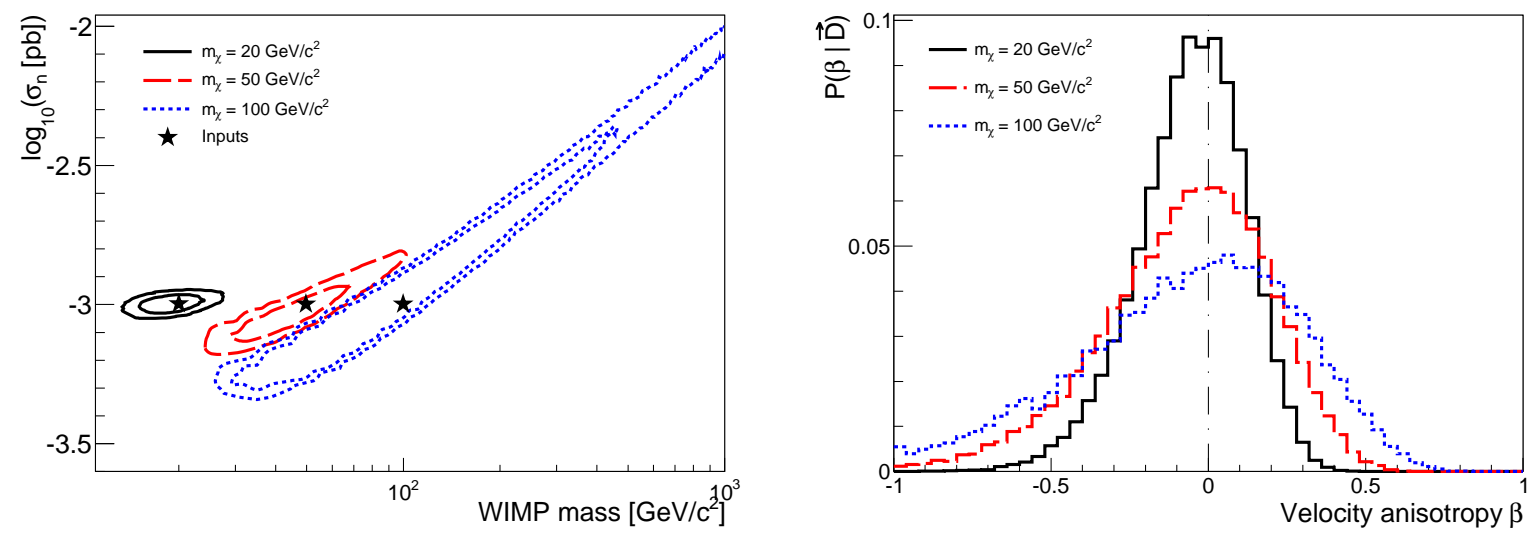

FIG. 7: Left panel : $68 \%$ and $95 \%$ contour level in the $\left(m_{\chi}, \sigma_{n}\right)$ plane, for the isotropic input model and for a WIMP mass equal to 20, 50 and $100 \mathrm{GeV} / \mathrm{c}^{2}$. Right panel : posterior PDF distribution of the $\beta$ parameter for the same models.
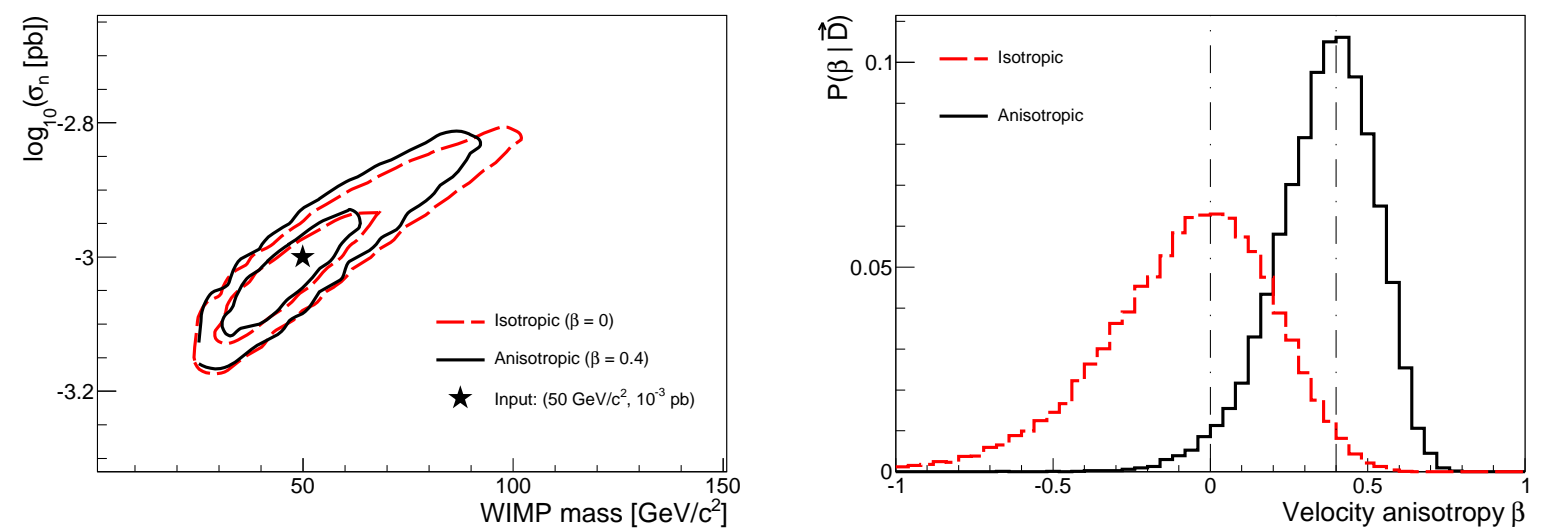

FIG. 8: Left panel : $68 \%$ and $95 \%$ contour level in the $\left(m_{\chi}, \sigma_{n}\right)$ plane, for a $50 \mathrm{GeV} / \mathrm{c}^{2}$ WIMP and for two input models : isotropic $(\beta=0)$ and triaxial $(\beta=0.4)$. Right panel : posterior PDF distribution of the $\beta$ parameter for the same models.
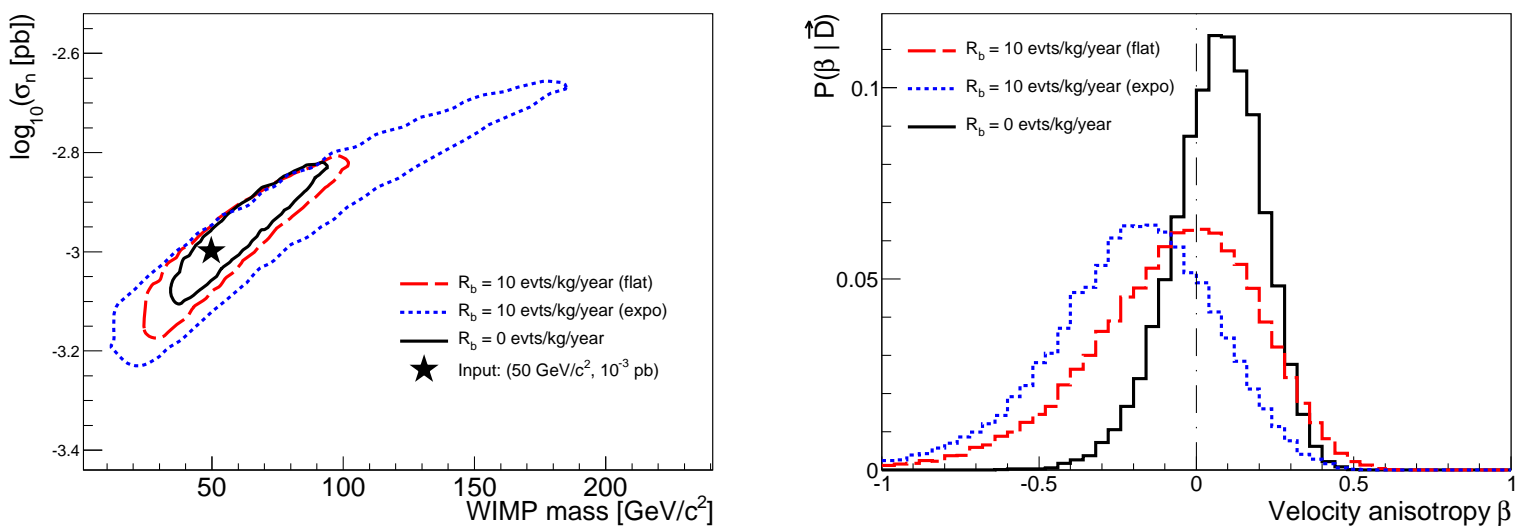

FIG. 9: Left panel : 95\% contour level in the $\left(m_{\chi}, \sigma_{n}\right)$ plane, for a $50 \mathrm{GeV} / \mathrm{c}^{2}$ WIMP and for three input background model : no background, flat spectrum and exponential spectrum. Right panel : posterior PDF distribution of the $\beta$ parameter for the same models. 


\begin{tabular}{|c|c|c|c|c|}
\hline Halo & Background & $m_{\chi}\left(\mathrm{GeV} / \mathrm{c}^{2}\right)$ & $\beta_{\text {in }}$ & $\beta_{\text {out }}$ \\
\hline \hline Isotropic & Flat & 20 & 0 & $-0.06_{-0.1}^{+0.2}$ \\
\hline Isotropic & Flat & 50 & 0 & $-0.07_{-0.2}^{+0.3}$ \\
\hline Isotropic & Expo. & 50 & 0 & $-0.20_{-0.2}^{+0.3}$ \\
\hline Isotropic & NO & 50 & 0 & $+0.05_{-0.1}^{+0.1}$ \\
\hline Isotropic & Flat & 100 & 0 & $-0.10_{-0.2}^{+0.4}$ \\
\hline \hline Anisotropic & Flat & 50 & 0.4 & $+0.38_{-0.1}^{+0.2}$ \\
\hline
\end{tabular}

TABLE III: Values of the $\beta$ parameter from the marginalized distribution for various input models. We quote mean value of the PDF distribution and $68 \%$ CL error bars.

for the three WIMP masses, in the left panel, the $68 \%$ and $95 \%$ CL contours in the $\left(m_{\chi}, \log _{10}\left(\sigma_{n}\right)\right)$ plane, and in the right panel, the posterior $\operatorname{PDF} P(\beta \mid \vec{D})$ of the anisotropy velocity parameter $\beta$.

The WIMP properties $\left(m_{\chi}, \log _{10}\left(\sigma_{n}\right)\right)$ are consistently constrained according to the input values with no $a$ priori knowledge of the halo properties, as the velocity dispersions are set as free parameters of the analysis. It can be deduced from Fig. 7 that this analysis is working for any input WIMP mass even if the constraints strongly depend on the input value. Indeed, as it can be seen in Fig. 7, the constraints on $\left(m_{\chi}, \log _{10}\left(\sigma_{n}\right)\right)$ are very tight below $50 \mathrm{GeV} / c^{2}$ and become wider for increasing WIMP mass.

In fact, for the $100 \mathrm{GeV} / \mathrm{c}^{2}$ input WIMP mass, only a lower limit should be deduced as $m_{\chi}>30 \mathrm{GeV} / \mathrm{c}^{2}$ (68\% C.L.). Indeed, the $68 \%$ and $95 \%$ C.L. contours correspond to the case where a flat prior on $m_{\chi} \in\left[5,10^{3}\right]$ $\mathrm{GeV} / \mathrm{c}^{2}$ is considered. These weaker constraints in the case of a heavy WIMP are due to the fact that the signal characteristics, i.e the slope of the energy distribution and the width of the angular distribution, evolve slowly with the WIMP mass once $m_{\chi} \geq 100 \mathrm{GeV} / \mathrm{c}^{2}$ for a fluorine target and a recoil energy in the range $[5,50]$ $\mathrm{keV}$, as shown in [8].

As a consequence of this weaker constraint at heavy WIMP masses, the constraints on the halo properties are also getting weaker, albeit with smaller effect. Indeed, as shown in the right panel of Fig. 7 the constraint on the anisotropy velocity parameter $\beta$ is stronger (smaller error bars) for an input WIMP mass of $20 \mathrm{GeV} / \mathrm{c}^{2}$ than for a $100 \mathrm{GeV} / \mathrm{c}^{2}$ one. However, as highlighted in Table III. the constraint on the $\beta$ parameter remains competitive and for a $30 \mathrm{~kg}$.year exposure with a MIMAC like directional detector, this MCMC would allow us to get, in this case, a strong evidence in favor of an isotropic dark matter halo.

\section{B. Effect of an anisotropic input halo model}

In this section, we vary the input halo model to evaluate the evolution of the constraints associated with the different dark matter properties $\left(m_{\chi}, \sigma_{n}, \beta\right)$. Indeed, as the velocity dispersions are set as free parameters, induced bias due to wrong model assumption should be avoided. This is for instance the effect observed in [13], with a systematic downward shift of the estimated cross section, when assuming a standard isotropic velocity distribution fitting model whereas the input model is a triaxial one.

In the following, we investigate the effect of an extremely triaxial input halo model with $\beta=0.4$ (see sec II) on the estimation of the dark matter parameters.

The results from the MCMC run on a simulated dataset corresponding to a WIMP mass of $50 \mathrm{GeV} / \mathrm{c}^{2}$ with the latter anisotropic halo model are presented in Fig. 8. As for the previous section, in the left panel is presented the constraint at $68 \%$ and $95 \%$ on the $\left(m_{\chi}, \log _{10}\left(\sigma_{n}\right)\right)$ plane, while in the right panel is given the deduced posterior PDF of the $\beta$ parameter. For convenience and comparison, the results from the benchmark input model (isothermal sphere with a 50 $\mathrm{GeV} / \mathrm{c}^{2}$ WIMP) are recalled.

From the left panel of Fig. 8, we can conclude that the two halo models give similar constraints which are both consistent with the input values. In fact, and as foreseen, the fact that the velocity dispersions are set as free parameters in the MCMC analysis allows us to avoid induced bias due to wrong model assumption.

From the right panel of Fig. 8 we can deduce that the $\beta$ parameter is well constrained: $\beta=0.38_{-0.1}^{+0.2}$, as in the isotropic case. In fact, the constraint is even stronger in the anisotropic case than in the isotropic one. This comes straightforwardly from the decrease of the degeneracy between the three velocity dispersions with increasing departure from isotropy.

As a conclusion of this study, it should be highlighted that the combination of information from the angular and energy distributions leads to robust allowed regions in the $\left(m_{\chi}, \log _{10}\left(\sigma_{n}\right)\right)$ plane, since the halo model is also being constrained with the MCMC analysis from the same dataset of a single directional detection experiment. Moreover, the velocity anisotropy parameter $\beta$, i.e. the three velocity dispersions, could be sufficiently constrained to discriminate between different halo models with future directional detectors such as the one proposed by the MIMAC collaboration [3].

\section{Varying the input background spectrum}

The background energy spectrum is a key issue for both directional detection and direct detection (direction-insensitive experiments). When setting ex- 


\begin{tabular}{|c|c|c|c|}
\hline Halo & Background & $R_{b}^{i n}$ & $R_{b}$ MCMC output \\
\hline \hline Isotropic & Flat & 10 & $10.97_{-1}^{+1}$ \\
\hline Isotropic & Expo. & 10 & $10.03_{-2}^{+3}$ \\
\hline Isotropic & NO & 0 & $<0.36$ (upper limit) \\
\hline \hline Anisotropic & Flat & 10 & $9.8_{-1}^{+1}$ \\
\hline
\end{tabular}

TABLE IV: Values of the $R_{b}$ parameter (in $\mathrm{kg}^{-1}$ year $^{-1}$ ) from the marginalized distribution for various background input models. We quote mean value of the PDF distribution while the error bars and upper limits are quoted with a $68 \%$ CL.

clusion limits with directional detection, the difficulty can be avoided by considering only the angular part of the directional event rate, thus allowing us to set robust and conservative limits [7]. But as far as the whole directional event rate is used, the question of the background energy spectrum must be carefully treated. In fact, as the background energy spectrum is unknown it must be guessed to be included in a likelihood-type analysis. Then, a wrong assumption on the background shape leads to an incorrect estimation of the background rate, resulting in a wrong estimation of the dark matter properties.

Motivated by simulations of neutron background in dark matter detectors, e.g. in low pressure TPC [51], two different background energy distributions are usually considered [12 17] : flat and exponentially decreasing with increasing recoil energy. The exponential one corresponds to the most pessimistic case as it is chosen, in our case, to be exactly the same as the WIMP-induced energy distribution. That is to say an exponential distribution with a slope of $\sim 17 \mathrm{keV}$ in the case where the WIMP mass is $50 \mathrm{GeV} / \mathrm{c}^{2}$ and considering an isotropic halo model. As outlined in [14], it is not possible to disentangle a WIMP signal from the background, with a single direct detector, if the shape of the background and WIMP-induced energy distributions are similar. In principle, this will not be the case for directional detection as the angular distribution of the background is isotropic, then remaining different from the WIMP-induced one.

Figure 9presents the constraints in the $\left(m_{\chi}, \log _{10}\left(\sigma_{n}\right)\right)$ plane and on the $\beta$ parameter for three input background energy distributions: no background (black solid line), flat (red dashed line) and exponential (blue dotted line). The other input parameters are those used in Sec. V for the benchmark input model (an isotropic halo, a $50 \mathrm{GeV} / \mathrm{c}^{2}$ WIMP mass and a $10^{-3} \mathrm{pb}$ WIMP-nucleon axial cross section).

First, the comparison between the case with no background and the case with a flat background energy distribution highlights the fact that even with a large background contamination $(\sim 30 \%)$, the results are quite similar, particularly in the determination of the WIMP properties, due to the fact that the disentanglement between WIMP and background events is done with both energy and directional arguments. Then, in both cases, as the background rate is correctly estimated by the MCMC analysis (see Table IV], systematic bias in the estimation of the dark matter properties is avoided. However, from Fig. 9 and Table III, it should be noticed that, even if the $\beta$ parameter is consistently constrained according to the input value, the presence of a sizeable background leads to a wider constraint (about two times larger).

In the case of an exponential input background energy distribution, the result is basically unchanged, although constraints are weaker. This is due to the fact that the background event rate parameter is less constrained (see Table [V] resulting in broader marginalized distributions of other parameters. Indeed, the estimation of the $R_{b}$ parameter is done solely with the angular part of the spectrum, as the energy distributions are exactly the same for both kinds of events. Nevertheless, even in such a pessimistic case, the WIMP properties $\left(m_{\chi}, \log _{10}\left(\sigma_{n}\right)\right)$ and the dark matter halo properties encoded in the $\beta$ parameter can still be estimated with upcoming directional detectors with realistic exposures thanks to the use of this MCMC analysis.

From this study, it should be concluded that, the effect of background contamination on directional data can be handled in the case where the background energy distribution is correctly estimated. Eventually, we have shown that even for a large background contamination and in the most pessimistic background model, directional detection combined with this MCMC analysis should allow us to assess consistent and interesting constraints on the dark matter properties with a single experiment.

\section{CONCLUSION}

We have shown that identification of dark matter might be achieved with a $10 \mathrm{~kg} \mathrm{CF}_{4}$ directional detector, allowing $3 \mathrm{D}$ track reconstruction with sense recognition down to $5 \mathrm{keV}$ and operated during three years. To fully exploit upcoming data, we propose a new high dimensional multivariate analysis method based on a Markov chain Monte Carlo analysis of recoil events, allowing to constrain, in a single directional experiment, the WIMP parameters, both from particle physics (mass and cross section) and Galactic halo (velocity dispersion along the three axis) and within the framework of a given ansatz.

Indeed, the combination of information from the angular and energy distributions leads to robust allowed regions in the $\left(m_{\chi}, \log _{10}\left(\sigma_{n}\right)\right)$ plane, since the halo model is also being constrained with the MCMC analysis from the same dataset of a single directional detection experiment. Moreover, the velocity anisotropy parameter $\beta$, related to the three velocity dispersions, could be suffi- 
ciently constrained to discriminate between various halo models with future directional detectors such as the one proposed by the MIMAC collaboration [3].

\section{Acknowledgements}

The authors would like to thank Antje Putze and Laurent Derome for fruitful discussions concerning MCMC methods.
[1] D. N. Spergel, Phys. Rev. D 37 (1988) 1353

[2] S. Ahlen et al., Int. J. Mod. Phys. A 25 (2010) 1

[3] D. Santos et al., arXiv:1012.1166

[4] E. Daw et al., arXiv:1010.3027 [astro-ph.CO].

[5] S. Ahlen et al., Phys. Lett. B695 (2011) 124-129

[6] K. Miuchi et al., Phys. Lett. B 686 (2010) 11

[7] J. Billard, F. Mayet and D. Santos, Phys. Rev. D 82 (2010) 055011

[8] J. Billard, F. Mayet, J. F. Macias-Perez and D. Santos, Phys. Lett. B 691 (2010) 156-162

[9] A. M. Green and B. Morgan, Phys. Rev. D 81 (2010) 061301

[10] J. Billard, F. Mayet and D. Santos, arXiv:1009.5568. [Proc. Sci. (to be published)].

[11] N. Bernal and S. Palomares-Ruiz, arXiv:1006.0477

[12] N. Bernal, A. Goudelis, Y. Mambrini and C. Munoz, JCAP 0901 (2009) 046

[13] A. M. Green, JCAP 0708 (2007) 022

[14] A. M. Green, JCAP 0807 (2008) 005

[15] M. Drees and C. L. Shan, JCAP 0706 (2007) 011

[16] M. Drees and C. L. Shan, JCAP 0806 (2008) 012

[17] C. L. Shan, Mod. Phys. Lett. A 25 (2010) 951

[18] J. Billard et al., in preparation

[19] G. Bertone et al., Phys. Rev. D 82 (2010) 055008

[20] L. E. Strigari and R. Trotta, JCAP 0911 (2009) 019

[21] M. S. Alenazi and P. Gondolo, Phys. Rev. D 77 (2008) 043532

[22] A. M. Green, arXiv:1009.0916

[23] P. D. Serpico and G. Bertone, Phys. Rev. D 82 (2010) 063505

[24] F. S. Ling, Phys. Rev. D 82 (2010) 023534

[25] F. S. Ling, E. Nezri, E. Athanassoula and R. Teyssier, JCAP 1002 (2010) 012

[26] T. Bruch, J. Read, L. Baudis and G. Lake, Astrophys. J. 696 (2009) 920

[27] J. I. Read, G. Lake, O. Agertz and V. P. Debattista, MNRAS 389 (2008) 1041-1057

[28] D. R. Law, S. R. Majewski and K. V. Johnston, Astrophys. J. 703 (2009) L67

[29] E. Hayashi, J. F. Navarro and V. Springel, Mon. Not.
Roy. Astron. Soc. 377 (2007) 50

[30] N. W. Evans, C. M. Carollo and P. T. de Zeeuw, Mon. Not. Roy. Astron. Soc. 318 (2000) 1131 arXiv:astro-ph/0008156.

[31] J. Binney and S. Tremaine, Galactic dynamics, Princeton University Press (2008)

[32] M. Vogelsberger et al., Monthly Notices of the Royal Astronomical Society 395 (2009) 797-811

[33] M. Kuhlen et al., JCAP 1002 (2010) 030

[34] B. Moore, C. Calcaneo-Roldan, J. Stadel, T. R. Quinn, G. Lake, S. Ghigna and F. Governato, Phys. Rev. D 64 (2001) 063508

[35] R. Teyssier, Astron. Astrophys. 385 (2002) 337

[36] C. Golabek et al., in preparation

[37] P. Gondolo, Phys. Rev. D 66 (2002) 103513

[38] J. D. Lewin \& P. F. Smith, Astropart. Physics 6 (1996) $87-112$

[39] J. Radon, Ber. Verh. Schs. Akad. Wiss. Leipzig, Math. Nat. kl. 69 (1917) 262-277.

[40] C. J. Copi, L. M. Krauss Phys. Rev. D 63 (2001) 043507

[41] C. Grignon et al., JINST 4 (2009) P11003

[42] A. Putze et al., Astron. Astrophys. 497 (2009) 991-1007

[43] V. L. Corless and L. J. King, Mon. Not. Roy. Astron. Soc. 390 (2008) 997-1013

[44] G. Cowan, Statistical Data Analysis, Clarendon Press, Oxford, 1998.

[45] Y. T. Chou and C. L. Shan, JCAP 1008 (2010) 014

[46] C. L. Shan, JCAP 1006 (2010) 029

[47] C. L. Shan, New J. Phys. 11 (2009) 105013

[48] K. Nakamura et al. (Particle Data Group), Journal of Physics G37 (2010) 075021

[49] P. Salucci, F. Nesti, G. Gentile and C. F. Martins, arXiv:1003.3101

[50] D. Mei and A. Hime, Phys. Rev. D 73 (2006) 053004

[51] M. J. Carson et al., Nucl. Instrum. Meth. A 546 (2005) 509 arXiv:hep-ex/0503017.

[52] Obviously, for real data analysis, these two components of the detector velocity have to be considered in order to have an accurate analysis. 